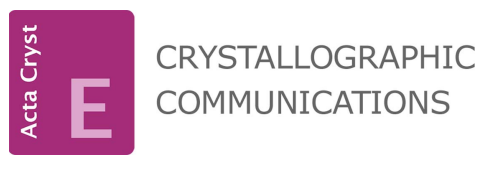

ISSN 2056-9890

Received 4 March 2015

Accepted 5 May 2015

Edited by S. Parkin, University of Kentucky, USA

Keywords: crystal structure; 3-amino-4-nitrobenzyl acetate; intramolecular; intermolecular; resonance-assisted hydrogen bonding; 5-amino2-nitrobenzoic acid

CCDC reference: 1063364 Supporting information: this article has supporting information at journals.iucr.org/e

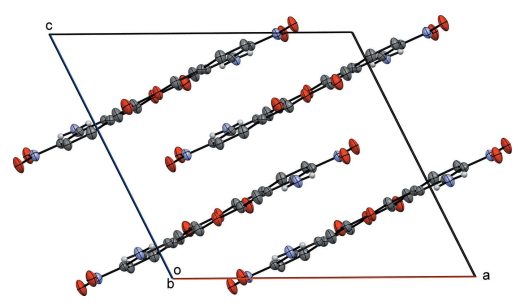

OPEN $\odot$ ACCESS

\section{Isolation of 3-amino-4-nitrobenzyl acetate: evidence of an undisclosed impurity in 5-amino-2-nitrobenzoic acid}

\author{
Brandon Quillian,* Jordan Hendricks, Matthew Trivitayakhun and Clifford W. \\ Padgett
}

Department of Chemistry and Physics, Armstrong State University, 11935 Abercorn Street, Savannah GA 31419, USA *Correspondence e-mail: Brandon.Quillian@armstrong.edu

Yellow crystals of the title compound 3-amino-4-nitrobenzyl acetate, $\mathrm{C}_{9} \mathrm{H}_{10} \mathrm{~N}_{2} \mathrm{O}_{4}$, were isolated from the reaction of acetic anhydride with (5amino-2-nitrophenyl)methanol, prepared from reduction of commerically available 5-amino-2-nitrobenzoic acid with borane-THF. The molecule is essentially planar (r.m.s. deviation $=0.028 \AA$ ). The molecules are linked by intermolecular $\mathrm{N}-\mathrm{H}$. . O hydrogen-bonding interactions between the carbonyl and amine groups, forming a zigzag chain along the $b$-axis direction lying in a plane parallel to (102). The chains are stacked along the $c$ axis by $\pi-\pi$ interactions [centroid-centroid distances $=3.6240$ (3) and 3.5855 (4) $\AA$ ]. A strong intramolecular $\mathrm{N}-\mathrm{H} \cdots \mathrm{O}$ hydrogen-bonding interaction is observed between the nitro group and the amine group [2.660 (2) ̊].

\section{Chemical Context}

Often commercially available chemicals are sold with minor impurities in the range $1-5 \%$; the user may choose to 'use as received' or further purify. The identities of the impurities are rarely disclosed in fine chemicals. Though these impurities may serve as benign spectators, in some cases they might hinder reactivity and/or produce undesirable by-products that are difficult to separate from the desired product. Therefore, it is important to identify these impurities to allow the users to decide if further purification is warranted. We recently purchased 5-amino-2-nitrobenzoic acid from Acros Organics ${ }^{\circledR}$ (5 g, 97\%, AC33074-0050) for our ongoing studies of photoinduced decarboxylation of ortho-nitrobenzyl esters (Cabane et al., 2010; Pocker et al., 1978). The isolation of the title compound, 3-amino-4-nitrobenzyl acetate, after the reaction of crude (5-amino-2-nitrophenyl)methanol, prepared from the reduction of 5-amino-2-nitrobenzoic acid, with acetic anhydride suggests 3-amino-4-nitrobenzoic acid is an impurity in the commercially available starting material.<smiles>CC(=O)OCc1ccc([N+](=O)[O-])c(N)c1</smiles>

\section{Structural Commentary}

The asymmetric unit of the title compound (Fig. 1) displays an essentially planar molecule (r.m.s.d. $0.028 \AA$ ) with the amine, nitro and acetate groups resting in the plane of the arene. The 


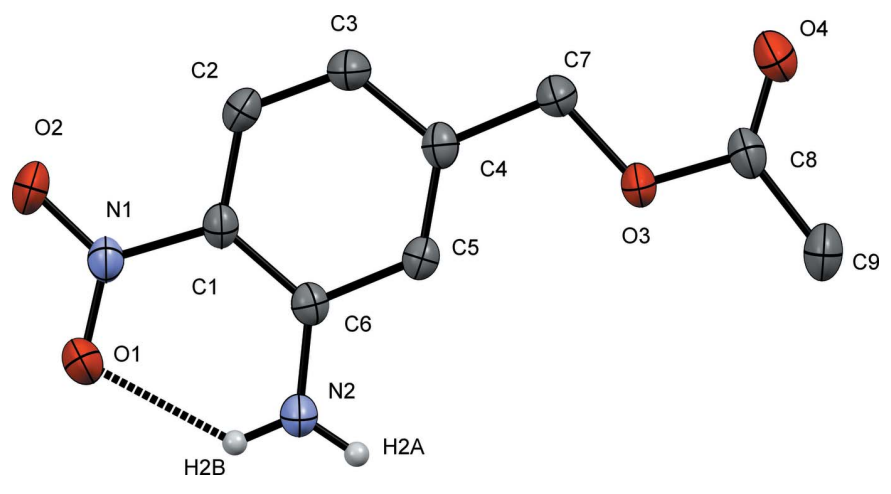

Figure 1

A displacement ellipsoid plot of 3-amino-4-nitrobenzyl acetate $(50 \%$ probability level). C-bound $\mathrm{H}$ atoms have been omitted for clarity.

carbonyl, $\quad \mathrm{C}=\mathrm{O} \quad[1.208(2) \AA], \quad$ and ester, $\quad \mathrm{C}-\mathrm{O}$ [1.3477 (19) §], bond distances are unassuming. The nitro bond distances $[\mathrm{O} 1-\mathrm{N} 1 \quad 1.2500(16)$ and $\mathrm{O} 2-\mathrm{N} 1$ $1.2401(17) \AA]$ are similar to those in $N$-(3-chlorophenyl)-3nitropyridin-2-amine [1.222 (2) and 1.245 (2) $\mathrm{A}]$ (Aznan et al., 2011). Atom O1 of the nitro group is involved in strong intramolecular hydrogen bonding [graph set $S 1,1(6)$ ] between $\mathrm{H} 2 B$ of the amine at a distance of 2.06 (2) $\AA$, forming a rigid, thermodynamically stable six-membered ring (Fig. 1). The elongated $\mathrm{O} 1-\mathrm{N} 1$ bond distance, as compared to the $\mathrm{O} 2-\mathrm{N} 1$ distance, is consistent with resonance-assisted hydrogen bonding between $\mathrm{O} 1$ and $\mathrm{H} 2 B$ (Beck \& Mo, 2006).

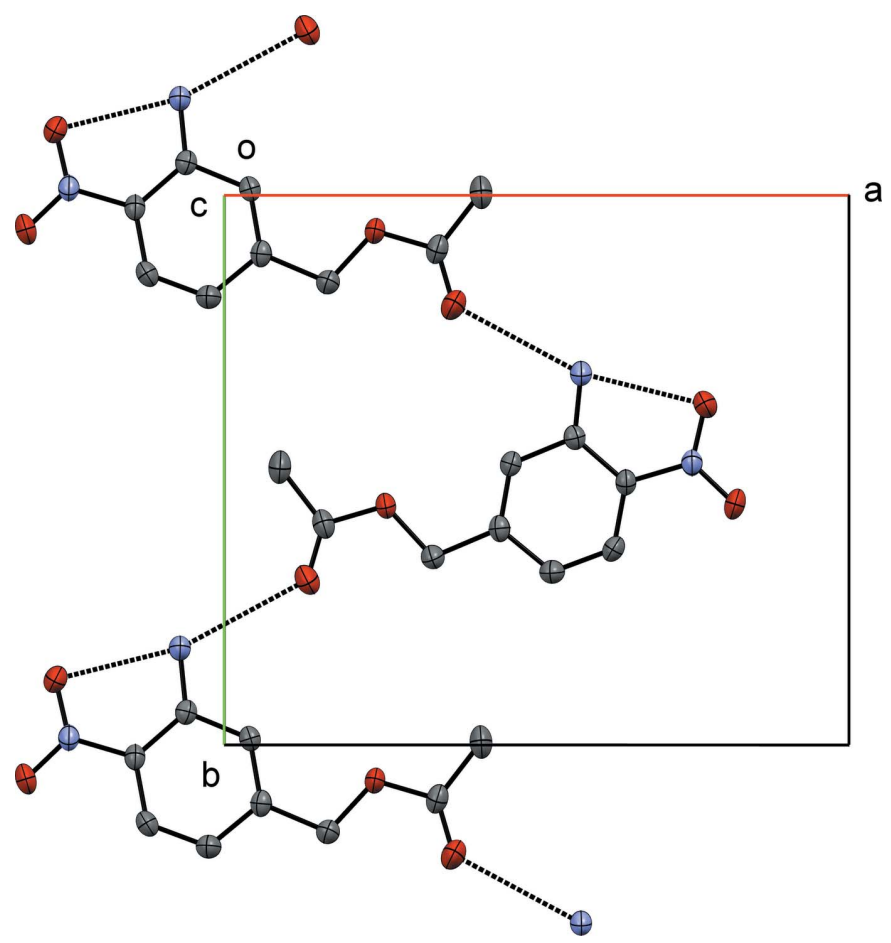

Figure 2

A single of layer of the unit cell of 3-amino-4-nitrobenzoic acid through the $a b$ plane (observed down the $c$ axis), highlighting the hydrogenbonding motif.
Table 1

Hydrogen-bond geometry $\left(\AA,^{\circ}\right)$.

\begin{tabular}{lclll}
\hline$D-\mathrm{H} \cdots A$ & $D-\mathrm{H}$ & $\mathrm{H} \cdots A$ & $D \cdots A$ & $D-\mathrm{H} \cdots A$ \\
\hline $\mathrm{N} 2-\mathrm{H} 2 A \cdots \mathrm{O} 4^{\mathrm{i}}$ & $0.83(2)$ & $2.18(2)$ & $3.005(2)$ & $171.5(17)$ \\
$\mathrm{N} 2-\mathrm{H} 2 B \cdots \mathrm{O} 1$ & $0.84(2)$ & $2.06(2)$ & $2.6600(19)$ & $128.0(16)$ \\
$\mathrm{N}^{\mathrm{i}}-\mathrm{H} 2 B \cdots \mathrm{O} 1^{\mathrm{ii}}$ & $0.84(2)$ & $2.44(2)$ & $3.1443(19)$ & $142.7(16)$ \\
\hline
\end{tabular}

Symmetry codes: (i) $-x+\frac{3}{2}, y+\frac{1}{2},-z+\frac{3}{2}$; (ii) $-x+\frac{1}{2},-y+\frac{3}{2},-z+1$.

\section{Supramolecular Features}

The crystal structure of 3-amino-4-nitrobenzyl acetate has interesting supramolecular features. The molecules are arranged in layers held together by intermolecular $\mathrm{N} 2-$ $\mathrm{H} 2 A \cdots \mathrm{O} 4[3.005$ (2) $\AA$ ] hydrogen bonding [graph set $C 1,1(9)$ ] interactions between the carbonyl and amine groups forming a zigzag chain along the $b$-axis direction (Fig. 2 and Table 1 ) lying in a plane parallel to (102). A view of a single layer along the $a b$ plane, observed down the $c$ axis (Fig. 2) provides a representative illustration of the hydrogen-bonding interactions of 3-amino-4-nitrobenzyl acetate. Observing the unit cell along the $b$-axis (Fig. 3) shows four layers along the $c$ axis separated at a distance of 3.3163 (10) $\AA$ with the arene groups stacked one above the other. The chains stack along the $c$ axis by $\pi-\pi$ interactions [centroid-centroid distances = 3.6240 (3) $\AA$ (symmetry code $1-x, 1-y, 1-z$ ) and 3.5855 (4) $\AA$ (symmetry code $1-x, y, \frac{3}{2}-z$ )].

\section{Database Survey}

For a related benzyl acetate structure, see Kasuga et al. (2015). For alkyl- and aryl-3-amino-4-nitro-benzoates and benzoic acids displaying similar intramolecular hydrogen bonding between the amino and nitro groups, see: Narendra Babu et al. (2009); Abdul Rahim et al. (2010); Yoon et al. (2011); Yoon et al. (2012).

\section{Synthesis and Crystallization}

(5-Amino-2-nitrophenyl)methanol: (5-amino-2-nitrophenyl)methanol was prepared by a modified literature protocol

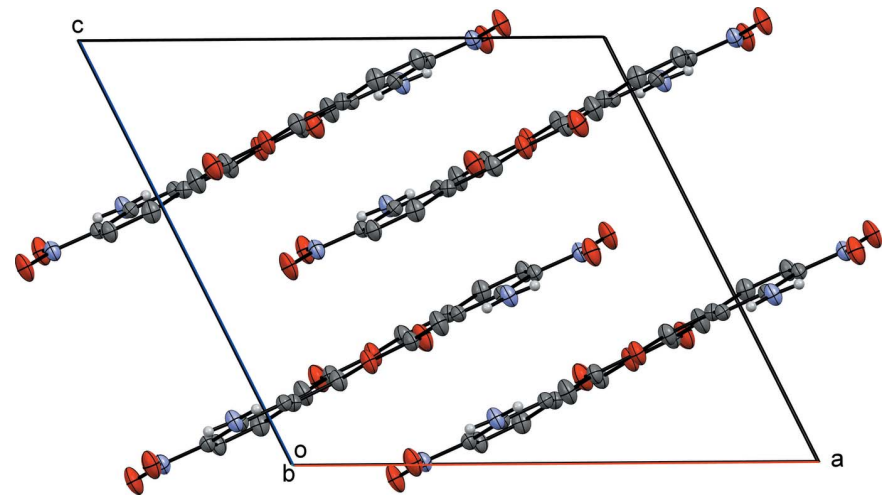

Figure 3

A displacement ellipsoid plot of the unit cell of 3-amino-4-nitrobenzoic acid observed down the $b$ axis. 
Table 2

Experimental details.

\begin{tabular}{|c|c|}
\hline Crystal data & \\
\hline Chemical formula & $\mathrm{C}_{9} \mathrm{H}_{10} \mathrm{~N}_{2} \mathrm{O}_{4}$ \\
\hline$M_{\mathrm{r}}$ & 210.19 \\
\hline Crystal system, space group & Monoclinic, $C 2 / c$ \\
\hline Temperature $(\mathrm{K})$ & 173 \\
\hline$a, b, c(\AA)$ & $\begin{array}{l}\text { 14.4803 (15), } 11.4054(11), \\
\quad 13.0936(13)\end{array}$ \\
\hline$\beta\left({ }^{\circ}\right)$ & $116.341(8)$ \\
\hline$V\left(\AA^{3}\right)$ & $1937.9(4)$ \\
\hline$Z$ & 8 \\
\hline Radiation type & Мo $K \alpha$ \\
\hline$\mu\left(\mathrm{mm}^{-1}\right)$ & 0.12 \\
\hline Crystal size $(\mathrm{mm})$ & $0.25 \times 0.25 \times 0.10$ \\
\hline Data collection & \\
\hline Diffractometer & Rigaku Mercury375R \\
\hline Absorption correction & $\begin{array}{l}\text { Multi-scan (REQAB; Rigaku, } \\
\text { 1998) }\end{array}$ \\
\hline$T_{\min }, T_{\max }$ & $0.840,1.000$ \\
\hline $\begin{array}{l}\text { No. of measured, independent and } \\
\text { observed }[I>2 \sigma(I)] \text { reflections }\end{array}$ & $8409,1759,1348$ \\
\hline$R_{\text {int }}$ & 0.045 \\
\hline$(\sin \theta / \lambda)_{\max }\left(\AA^{-1}\right)$ & 0.601 \\
\hline Refinement & \\
\hline$R\left[F^{2}>2 \sigma\left(F^{2}\right)\right], w R\left(F^{2}\right), S$ & $0.037,0.098,1.06$ \\
\hline No. of reflections & 1759 \\
\hline No. of parameters & 176 \\
\hline $\mathrm{H}$-atom treatment & All $\mathrm{H}$-atom parameters refined \\
\hline$\Delta \rho_{\max }, \Delta \rho_{\min }\left(\mathrm{e} \AA^{-3}\right)$ & $0.21,-0.17$ \\
\hline
\end{tabular}

Computer programs: CrystalClear-SM Expert (Rigaku, 2014), SHELXT (Sheldrick, 2015a), SHELXL2013 (Sheldrick, 2015b) and OLEX2 (Dolomanov et al., 2009).

(Yoon et al. 1973). To a solution of 5-amino-2-nitrobenzoic acid $(97 \%, 1.5 \mathrm{~g}, 8.2 \mathrm{mmol})$ dissolved in tetrahydrofuran (10 mL), borane-THF (27.6 mL, $1.0 \mathrm{M}$ in THF, $27.6 \mathrm{mmol})$ was added dropwise by dropping funnel over 30 minutes. The reaction was stirred overnight at room temperature. The reaction was quenched with aqueous potassium hydroxide $(2.45 M)$ until $\mathrm{pH} 11$ was reached and continued to be stirred for $6 \mathrm{~h}$, resulting in a greenish-brown solution. The solution was treated with a saturated solution of potassium carbonate followed by treatment with hydrochloric acid until $\mathrm{pH} 1$ was reached. The reaction mixture was extracted with diethyl ether three times; organic portions were collected and dried with anhydrous sodium sulfate overnight. The solution was filtered under vacuum, the filtrate was collected and all solvent removed under rotary evaporation to give a green powder $(0.68 \mathrm{~g}, 49 \%) .{ }^{1} \mathrm{H} \mathrm{NMR},\left(300 \mathrm{MHz}\right.$, acetone- $\left.d_{6}\right) \delta: 4.61(t, 1 \mathrm{H}$, $\left.-\mathrm{OH},{ }^{3} J_{\mathrm{HH}}=5.3 \mathrm{~Hz}\right), 4.95\left(d, 2 \mathrm{H}, \mathrm{CH}_{2},{ }^{3} J_{\mathrm{HH}}=5.3 \mathrm{~Hz}\right), 6.03$ (bs, 2H, $\left.\mathrm{NH}_{2}\right), 6.63\left(d \mathrm{~d}, 1 \mathrm{H}, \mathrm{Ar}-\mathrm{H},{ }^{3} J_{\mathrm{HH}}=8.8 \mathrm{~Hz},{ }^{3} J_{\mathrm{HH}}=\right.$ $2.3 \mathrm{~Hz}), 7.07(m, 1 \mathrm{H}, \mathrm{Ar}-\mathrm{H}), 8.02\left(d d, 1 \mathrm{H},{ }^{3} J_{\mathrm{HH}}=9.4 \mathrm{~Hz},{ }^{3} J_{\mathrm{HH}}\right.$ $=3.0 \mathrm{~Hz}$ ) (Aujard et al. 2006). Note: minor impurities were observed in the base line in the aromatic region.

3-Amino-4-nitrobenzyl acetate: (5-amino-2-nitrophenyl)methanol $(10 \mathrm{mg}, 0.0595 \mathrm{mmol})$ and triethylamine $(17 \mu \mathrm{L}$, $0.119 \mathrm{mmol})$ were dissolved in acetonitrile- $d_{6}(0.7 \mathrm{~mL})$ and added to an NMR tube. Acetic anhydride $(11.2 \mu \mathrm{L}$, $0.119 \mathrm{mmol}$ ) was added to the tube via a syringe. The tube was held at room temperature overnight. On completion of the reaction the solvent was removed in vacuo and the residue was reconstituted in a minimum amount of methylene chloride. The sample was loaded on a column of silica and eluted with an ethyl acetate/hexane solution $(70 / 30 v / v \%)$. The separated solutions were allowed to slowly evaporate at room temperature. The parent compound (5-amino-2-nitrobenzyl acetate) elutes first and is isolated as a yellow powder. ${ }^{1} \mathrm{H}$ NMR $\left(300 \mathrm{MHz}, \mathrm{CDCl}_{3}\right) \delta: 2.10\left(s, 3 \mathrm{H}, \mathrm{CH}_{3}\right), 4.35(\mathrm{bs}, 2 \mathrm{H}$, $\left.\mathrm{N} H_{2}\right), 5.50\left(s, 2 \mathrm{H}, \mathrm{CH}_{2}\right), 6.55\left(d d, 1 \mathrm{H}, \mathrm{Ar}-\mathrm{H},{ }^{3} J_{\mathbf{H H}}=8.9 \mathrm{~Hz}\right.$, $\left.{ }^{5} J_{\mathbf{H H}}=2.5 \mathrm{~Hz}\right), 6.68(m, 1 \mathrm{H}, \mathrm{Ar}-\mathrm{H}), 8.09\left(d d, 1 \mathrm{H}, \mathrm{Ar}-\mathrm{H},{ }^{3} J_{\mathbf{H H}}=\right.$ $8.9 \mathrm{~Hz},{ }^{\mathbf{5}} J_{\mathbf{H H}}=2.5 \mathrm{~Hz}$ ) (Serafinowski et al. 2008). Yellow crystals of the title compound were isolated (less than $1 \mathrm{mg}$ ) in later eluate. ${ }^{1} \mathrm{H}$ NMR $\left(300 \mathrm{MHz}, \mathrm{CDCl}_{3}\right) \delta: 2.19\left(s, 3 \mathrm{H}, \mathrm{CH}_{3}\right)$, $5.53\left(s, 2 \mathrm{H}, \mathrm{CH}_{2}\right), 7.44\left(b s, 2 \mathrm{H}, \mathrm{N} H_{2}\right), 7.65\left(d d, 1 \mathrm{H}, \mathrm{Ar}-\mathrm{H},{ }^{3} J_{\mathbf{H H}}\right.$ $\left.=8.9 \mathrm{~Hz},{ }^{5} J_{\mathbf{H H}}=2.5 \mathrm{~Hz}\right), 7.75(\mathrm{~m}, 1 \mathrm{H}, \mathrm{Ar}-\mathrm{H}), 8.15(d, 1 \mathrm{H}, \mathrm{Ar}-$ $\left.\mathrm{H},{ }^{3} J_{\mathbf{H H}}=8.9 \mathrm{~Hz}\right)$.

\subsection{Refinement}

Crystal data, data collection and structure refinement details are summarized in Table 2. Hydrogen atoms were refined freely.

\section{Acknowledgements}

Acknowledgments are made to Armstrong State University and to the Donors of the American Chemical Society Petroleum Research Fund for support (or partial support) of this research (PRF No. 53848-UNI3). Additional support was provided by the NSF-STEP Program under Award No. DUE0856593.

\section{References}

Abdul Rahim, A. S., Abd Hamid, S., Narendra Babu, S. N., Loh, W.-S. \& Fun, H.-K. (2010). Acta Cryst. E66, o846-0847.

Aujard, I., Benbrahim, C., Gouget, M., Ruel, O., Baudin, J.-B., Neveu, P. \& Jullien, L. (2006). Chem. Eur. J. 12, 6865-6879.

Aznan, A. M. A., Abdullah, Z., Ng, S. W. \& Tiekink, E. R. T. (2011). Acta Cryst. E67, o3076.

Beck, J. F. \& Mo, Y. (2006). J. Comput. Chem. 4, 455-466.

Cabane, E., Malinova, V. \& Meier, W. (2010). Macromol. Chem. Phys. 211, 1847-1856.

Dolomanov, O. V., Bourhis, L. J., Gildea, R. J., Howard, J. A. K. \& Puschmann, H. (2009). J. Appl. Cryst. 42, 339-341.

Kasuga, N. C., Saito, Y., Sato, H. \& Yamaguchi, K. (2015). Acta Cryst. E71, 483-486.

Narendra Babu, S. N., Abdul Rahim, A. S., Abd Hamid, S., Balasubramani, K. \& Fun, H.-K. (2009). Acta Cryst. E65, o2070o2071.

Pocker, Y., Davison, B. L. \& Deits, T. L. (1978). J. Am. Chem. Soc. 100, 3564-3567.

Rigaku (1998). REQAB. Rigaku Corporation, Tokyo, Japan.

Serafinowski, P. J. \& Garland, P. B. (2008). Org. Biomol. Chem. 6, 3284-3291.

Sheldrick, G. M. (2015a). Acta Cryst. A71, 3-8.

Sheldrick, G. M. (2015b). Acta Cryst. C71, 3-8.

Yoon, Y. K., Ali, M. A., Choon, T. S., Loh, W.-S. \& Fun, H.-K. (2011). Acta Cryst. E67, o2606.

Yoon, Y. K., Manogaran, E., Ali, M. A., Arshad, S. \& Razak, I. A. (2012). Acta Cryst. E68, o1684.

Yoon, N. M., Pak, C. S., Krishnamurthy, S. \& Stocky, T. P. (1973). J. Org. Chem. 38, 2786-2792. 


\section{supporting information}

Acta Cryst. (2015). E71, 606-608 [doi:10.1107/S2056989015008750]

\section{Isolation of 3-amino-4-nitrobenzyl acetate: evidence of an undisclosed impurity in 5-amino-2-nitrobenzoic acid}

Brandon Quillian, Jordan Hendricks, Matthew Trivitayakhun and Clifford W. Padgett

\section{Computing details}

Data collection: CrystalClear-SM Expert (Rigaku, 2014); cell refinement: CrystalClear-SM Expert (Rigaku, 2014); data reduction: CrystalClear-SM Expert (Rigaku, 2014); program(s) used to solve structure: SHELXT (Sheldrick, 2015a); program(s) used to refine structure: SHELXL2013 (Sheldrick, 2015b); molecular graphics: OLEX2 (Dolomanov et al., 2009); software used to prepare material for publication: OLEX2 (Dolomanov et al., 2009).

\section{3-Amino-4-nitrobenzyl acetate}

Crystal data

$\mathrm{C}_{9} \mathrm{H}_{10} \mathrm{~N}_{2} \mathrm{O}_{4}$

$M_{r}=210.19$

Monoclinic, $C 2 / c$

$a=14.4803(15) \AA$

$b=11.4054(11) \AA$

$c=13.0936(13) \AA$

$\beta=116.341(8)^{\circ}$

$V=1937.9(4) \AA^{3}$

$Z=8$

\section{Data collection}

Rigaku Mercury375R (2x2 bin mode) diffractometer

Radiation source: Sealed Tube

Graphite Monochromator monochromator

Detector resolution: 13.6612 pixels $\mathrm{mm}^{-1}$

profile data from $\omega$ scans

Absorption correction: multi-scan

(REQAB; Rigaku, 1998)

$T_{\min }=0.840, T_{\max }=1.000$

Refinement

Refinement on $F^{2}$

Least-squares matrix: full

$R\left[F^{2}>2 \sigma\left(F^{2}\right)\right]=0.037$

$w R\left(F^{2}\right)=0.098$

$S=1.06$

1759 reflections

176 parameters

0 restraints
$F(000)=880$

$D_{\mathrm{x}}=1.441 \mathrm{Mg} \mathrm{m}^{-3}$

Mo $K \alpha$ radiation, $\lambda=0.71075 \AA$

Cell parameters from 513 reflections

$\theta=1.6-25.4^{\circ}$

$\mu=0.12 \mathrm{~mm}^{-1}$

$T=173 \mathrm{~K}$

Prism, yellow

$0.25 \times 0.25 \times 0.10 \mathrm{~mm}$

8409 measured reflections

1759 independent reflections

1348 reflections with $I>2 \sigma(I)$

$R_{\text {int }}=0.045$

$\theta_{\max }=25.3^{\circ}, \theta_{\min }=2.4^{\circ}$

$h=-17 \rightarrow 17$

$k=-13 \rightarrow 13$

$l=-15 \rightarrow 15$

Primary atom site location: structure-invariant direct methods

Hydrogen site location: difference Fourier map

All $\mathrm{H}$-atom parameters refined

$w=1 /\left[\sigma^{2}\left(F_{\mathrm{o}}^{2}\right)+(0.0578 P)^{2}+0.2118 P\right]$

where $P=\left(F_{\mathrm{o}}^{2}+2 F_{\mathrm{c}}^{2}\right) / 3$

$(\Delta / \sigma)_{\max }<0.001$

$\Delta \rho_{\max }=0.21 \mathrm{e} \AA^{-3}$

$\Delta \rho_{\min }=-0.17$ e $\AA^{-3}$ 


\section{Special details}

Geometry. All e.s.d.'s (except the e.s.d. in the dihedral angle between two 1.s. planes) are estimated using the full covariance matrix. The cell e.s.d.'s are taken into account individually in the estimation of e.s.d.'s in distances, angles and torsion angles; correlations between e.s.d.'s in cell parameters are only used when they are defined by crystal symmetry. An approximate (isotropic) treatment of cell e.s.d.'s is used for estimating e.s.d.'s involving l.s. planes.

Fractional atomic coordinates and isotropic or equivalent isotropic displacement parameters $\left(\hat{A}^{2}\right)$

\begin{tabular}{lllll}
\hline & $x$ & $y$ & $z$ & $U_{\text {iso }} / U_{\text {eq }}$ \\
\hline O3 & $0.74138(8)$ & $0.43515(9)$ & $0.74195(10)$ & $0.0324(3)$ \\
O1 & $0.23008(8)$ & $0.61948(10)$ & $0.50492(11)$ & $0.0418(3)$ \\
O2 & $0.18245(8)$ & $0.43814(10)$ & $0.46692(11)$ & $0.0436(3)$ \\
O4 & $0.86707(9)$ & $0.30050(11)$ & $0.79735(12)$ & $0.0456(4)$ \\
N1 & $0.25141(9)$ & $0.51321(11)$ & $0.50551(11)$ & $0.0288(3)$ \\
N2 & $0.42892(12)$ & $0.67596(12)$ & $0.60612(12)$ & $0.0300(3)$ \\
H2A & $0.4821(16)$ & $0.7166(16)$ & $0.6337(16)$ & $0.036(5)^{*}$ \\
H2B & $0.3700(16)$ & $0.7046(15)$ & $0.5821(16)$ & $0.039(5)^{*}$ \\
C6 & $0.43920(11)$ & $0.55950(12)$ & $0.60073(12)$ & $0.0228(3)$ \\
C1 & $0.35704(10)$ & $0.47782(13)$ & $0.55271(12)$ & $0.0245(3)$ \\
C4 & $0.55919(11)$ & $0.39372(13)$ & $0.64720(12)$ & $0.0252(3)$ \\
C5 & $0.54112(10)$ & $0.51152(13)$ & $0.64747(11)$ & $0.0228(3)$ \\
H5 & $0.5978(13)$ & $0.5675(14)$ & $0.6814(13)$ & $0.024(4)^{*}$ \\
C2 & $0.37680(12)$ & $0.35678(14)$ & $0.54982(13)$ & $0.0295(4)$ \\
H2 & $0.3212(13)$ & $0.3041(14)$ & $0.5154(15)$ & $0.031(4)^{*}$ \\
C3 & $0.47512(12)$ & $0.31496(14)$ & $0.59587(14)$ & $0.0312(4)$ \\
H3 & $0.4895(13)$ & $0.2318(16)$ & $0.5930(15)$ & $0.033(4)^{*}$ \\
C7 & $0.66619(11)$ & $0.34199(14)$ & $0.69873(14)$ & $0.0296(4)$ \\
H7A & $0.6780(14)$ & $0.2874(16)$ & $0.7620(16)$ & $0.040(5)^{*}$ \\
H7B & $0.6772(12)$ & $0.2957(14)$ & $0.6407(15)$ & $0.032(4)^{*}$ \\
C8 & $0.84105(11)$ & $0.40198(15)$ & $0.79024(13)$ & $0.0307(4)$ \\
C9 & $0.91100(13)$ & $0.50537(18)$ & $0.83137(18)$ & $0.0419(5)$ \\
H9A & $0.8961(16)$ & $0.5562(19)$ & $0.7680(19)$ & $0.055(6)^{*}$ \\
H9B & $0.9809(17)$ & $0.4806(16)$ & $0.8703(17)$ & $0.046(5)^{*}$ \\
H9C & $0.8934(17)$ & $0.554(2)$ & $0.881(2)$ & $0.067(7)^{*}$ \\
& & & &
\end{tabular}

Atomic displacement parameters $\left(\AA^{2}\right)$

\begin{tabular}{lllllll}
\hline & $U^{11}$ & $U^{22}$ & $U^{33}$ & $U^{12}$ & $U^{13}$ & $U^{23}$ \\
\hline O3 & $0.0175(5)$ & $0.0288(6)$ & $0.0445(7)$ & $0.0021(4)$ & $0.0080(5)$ & $-0.0018(5)$ \\
O1 & $0.0242(6)$ & $0.0319(7)$ & $0.0621(8)$ & $0.0054(5)$ & $0.0128(6)$ & $0.0060(5)$ \\
O2 & $0.0194(6)$ & $0.0411(7)$ & $0.0601(8)$ & $-0.0073(5)$ & $0.0085(6)$ & $-0.0033(6)$ \\
O4 & $0.0271(6)$ & $0.0400(8)$ & $0.0605(8)$ & $0.0089(5)$ & $0.0111(6)$ & $-0.0009(6)$ \\
N1 & $0.0188(6)$ & $0.0314(8)$ & $0.0327(7)$ & $0.0002(5)$ & $0.0083(5)$ & $0.0031(6)$ \\
N2 & $0.0199(7)$ & $0.0271(8)$ & $0.0379(8)$ & $0.0002(6)$ & $0.0082(6)$ & $-0.0025(6)$ \\
C6 & $0.0214(7)$ & $0.0266(8)$ & $0.0210(7)$ & $0.0013(6)$ & $0.0098(6)$ & $0.0011(6)$ \\
C1 & $0.0180(7)$ & $0.0307(8)$ & $0.0234(7)$ & $0.0004(6)$ & $0.0078(6)$ & $0.0027(6)$ \\
C4 & $0.0210(7)$ & $0.0312(8)$ & $0.0239(7)$ & $0.0010(6)$ & $0.0104(6)$ & $0.0002(6)$ \\
C5 & $0.0198(8)$ & $0.0271(8)$ & $0.0212(7)$ & $-0.0025(6)$ & $0.0086(6)$ & $-0.0011(6)$
\end{tabular}


supporting information

\begin{tabular}{lllllll} 
C2 & $0.0218(8)$ & $0.0283(9)$ & $0.0358(9)$ & $-0.0063(7)$ & $0.0105(7)$ & $-0.0029(7)$ \\
C3 & $0.0279(8)$ & $0.0238(9)$ & $0.0406(9)$ & $-0.0004(6)$ & $0.0142(7)$ & $-0.0017(7)$ \\
C7 & $0.0237(8)$ & $0.0262(8)$ & $0.0363(9)$ & $0.0007(6)$ & $0.0110(7)$ & $-0.0024(7)$ \\
C8 & $0.0211(8)$ & $0.0379(10)$ & $0.0305(8)$ & $0.0056(7)$ & $0.0091(7)$ & $0.0011(7)$ \\
C9 & $0.0214(9)$ & $0.0483(12)$ & $0.0489(11)$ & $-0.0017(8)$ & $0.0091(8)$ & $-0.0015(9)$ \\
\hline
\end{tabular}

Geometric parameters $\left(\hat{A},{ }^{\circ}\right)$

\begin{tabular}{|c|c|c|c|}
\hline $\mathrm{O} 3-\mathrm{C} 7$ & $1.4449(19)$ & $\mathrm{C} 4-\mathrm{C} 3$ & $1.419(2)$ \\
\hline $\mathrm{O} 3-\mathrm{C} 8$ & $1.3477(19)$ & $\mathrm{C} 4-\mathrm{C} 7$ & $1.509(2)$ \\
\hline $\mathrm{O} 1-\mathrm{N} 1$ & $1.2500(16)$ & $\mathrm{C} 5-\mathrm{H} 5$ & $0.978(17)$ \\
\hline $\mathrm{O} 2-\mathrm{N} 1$ & $1.2401(17)$ & $\mathrm{C} 2-\mathrm{H} 2$ & $0.944(17)$ \\
\hline $\mathrm{O} 4-\mathrm{C} 8$ & $1.208(2)$ & $\mathrm{C} 2-\mathrm{C} 3$ & $1.362(2)$ \\
\hline $\mathrm{N} 1-\mathrm{C} 1$ & $1.4303(19)$ & $\mathrm{C} 3-\mathrm{H} 3$ & $0.975(18)$ \\
\hline $\mathrm{N} 2-\mathrm{H} 2 \mathrm{~A}$ & $0.83(2)$ & C7-H7A & $0.988(19)$ \\
\hline $\mathrm{N} 2-\mathrm{H} 2 \mathrm{~B}$ & $0.83(2)$ & $\mathrm{C} 7-\mathrm{H} 7 \mathrm{~B}$ & $0.994(17)$ \\
\hline $\mathrm{N} 2-\mathrm{C} 6$ & $1.342(2)$ & $\mathrm{C} 8-\mathrm{C} 9$ & $1.491(3)$ \\
\hline $\mathrm{C} 6-\mathrm{C} 1$ & $1.419(2)$ & C9-H9A & $0.96(2)$ \\
\hline $\mathrm{C} 6-\mathrm{C} 5$ & $1.4320(19)$ & C9-H9B & $0.95(2)$ \\
\hline $\mathrm{C} 1-\mathrm{C} 2$ & $1.414(2)$ & $\mathrm{C} 9-\mathrm{H} 9 \mathrm{C}$ & $0.97(2)$ \\
\hline $\mathrm{C} 4-\mathrm{C} 5$ & $1.369(2)$ & & \\
\hline $\mathrm{C} 8-\mathrm{O} 3-\mathrm{C} 7$ & $116.19(12)$ & $\mathrm{C} 3-\mathrm{C} 2-\mathrm{C} 1$ & $120.94(14)$ \\
\hline $\mathrm{O} 1-\mathrm{N} 1-\mathrm{C} 1$ & $119.36(12)$ & $\mathrm{C} 3-\mathrm{C} 2-\mathrm{H} 2$ & $119.5(10)$ \\
\hline $\mathrm{O} 2-\mathrm{N} 1-\mathrm{O} 1$ & $121.00(12)$ & $\mathrm{C} 4-\mathrm{C} 3-\mathrm{H} 3$ & $118.7(10)$ \\
\hline $\mathrm{O} 2-\mathrm{N} 1-\mathrm{C} 1$ & $119.64(13)$ & $\mathrm{C} 2-\mathrm{C} 3-\mathrm{C} 4$ & $119.75(15)$ \\
\hline $\mathrm{H} 2 \mathrm{~A}-\mathrm{N} 2-\mathrm{H} 2 \mathrm{~B}$ & $122.7(17)$ & $\mathrm{C} 2-\mathrm{C} 3-\mathrm{H} 3$ & $121.5(10)$ \\
\hline $\mathrm{C} 6-\mathrm{N} 2-\mathrm{H} 2 \mathrm{~A}$ & $118.1(12)$ & $\mathrm{O} 3-\mathrm{C} 7-\mathrm{C} 4$ & $109.47(13)$ \\
\hline $\mathrm{C} 6-\mathrm{N} 2-\mathrm{H} 2 \mathrm{~B}$ & $119.1(12)$ & $\mathrm{O} 3-\mathrm{C} 7-\mathrm{H} 7 \mathrm{~A}$ & $108.3(11)$ \\
\hline $\mathrm{N} 2-\mathrm{C} 6-\mathrm{C} 1$ & $125.60(13)$ & $\mathrm{O} 3-\mathrm{C} 7-\mathrm{H} 7 \mathrm{~B}$ & $109.9(9)$ \\
\hline $\mathrm{N} 2-\mathrm{C} 6-\mathrm{C} 5$ & $118.24(13)$ & $\mathrm{C} 4-\mathrm{C} 7-\mathrm{H} 7 \mathrm{~A}$ & $112.5(10)$ \\
\hline $\mathrm{C} 1-\mathrm{C} 6-\mathrm{C} 5$ & $116.16(13)$ & $\mathrm{C} 4-\mathrm{C} 7-\mathrm{H} 7 \mathrm{~B}$ & $110.3(10)$ \\
\hline $\mathrm{C} 6-\mathrm{C} 1-\mathrm{N} 1$ & $122.12(13)$ & $\mathrm{H} 7 \mathrm{~A}-\mathrm{C} 7-\mathrm{H} 7 \mathrm{~B}$ & $106.2(14)$ \\
\hline $\mathrm{C} 2-\mathrm{C} 1-\mathrm{N} 1$ & $117.02(13)$ & $\mathrm{O} 3-\mathrm{C} 8-\mathrm{C} 9$ & $111.22(14)$ \\
\hline $\mathrm{C} 2-\mathrm{C} 1-\mathrm{C} 6$ & $120.86(13)$ & $\mathrm{O} 4-\mathrm{C} 8-\mathrm{O} 3$ & $122.52(15)$ \\
\hline $\mathrm{C} 5-\mathrm{C} 4-\mathrm{C} 3$ & $119.84(14)$ & $\mathrm{O} 4-\mathrm{C} 8-\mathrm{C} 9$ & $126.26(15)$ \\
\hline $\mathrm{C} 5-\mathrm{C} 4-\mathrm{C} 7$ & $122.83(14)$ & $\mathrm{C} 8-\mathrm{C} 9-\mathrm{H} 9 \mathrm{~A}$ & $108.1(13)$ \\
\hline $\mathrm{C} 3-\mathrm{C} 4-\mathrm{C} 7$ & $117.33(14)$ & $\mathrm{C} 8-\mathrm{C} 9-\mathrm{H} 9 \mathrm{~B}$ & $110.5(11)$ \\
\hline $\mathrm{C} 6-\mathrm{C} 5-\mathrm{H} 5$ & $116.3(9)$ & $\mathrm{C} 8-\mathrm{C} 9-\mathrm{H} 9 \mathrm{C}$ & $110.8(13)$ \\
\hline $\mathrm{C} 4-\mathrm{C} 5-\mathrm{C} 6$ & $122.40(13)$ & $\mathrm{H} 9 \mathrm{~A}-\mathrm{C} 9-\mathrm{H} 9 \mathrm{~B}$ & $115.0(17)$ \\
\hline $\mathrm{C} 4-\mathrm{C} 5-\mathrm{H} 5$ & $121.3(9)$ & $\mathrm{H} 9 \mathrm{~A}-\mathrm{C} 9-\mathrm{H} 9 \mathrm{C}$ & $102.2(18)$ \\
\hline $\mathrm{C} 1-\mathrm{C} 2-\mathrm{H} 2$ & $119.6(10)$ & $\mathrm{H} 9 \mathrm{~B}-\mathrm{C} 9-\mathrm{H} 9 \mathrm{C}$ & $110.0(17)$ \\
\hline $\mathrm{O} 1-\mathrm{N} 1-\mathrm{C} 1-\mathrm{C} 6$ & $0.9(2)$ & $\mathrm{C} 5-\mathrm{C} 6-\mathrm{C} 1-\mathrm{N} 1$ & $178.48(13)$ \\
\hline $\mathrm{O} 1-\mathrm{N} 1-\mathrm{C} 1-\mathrm{C} 2$ & $-179.17(13)$ & $\mathrm{C} 5-\mathrm{C} 6-\mathrm{C} 1-\mathrm{C} 2$ & $-1.44(19)$ \\
\hline $\mathrm{O} 2-\mathrm{N} 1-\mathrm{C} 1-\mathrm{C} 6$ & $-178.35(13)$ & $\mathrm{C} 5-\mathrm{C} 4-\mathrm{C} 3-\mathrm{C} 2$ & $-1.7(2)$ \\
\hline $\mathrm{O} 2-\mathrm{N} 1-\mathrm{C} 1-\mathrm{C} 2$ & $1.6(2)$ & $\mathrm{C} 5-\mathrm{C} 4-\mathrm{C} 7-\mathrm{O} 3$ & $-2.0(2)$ \\
\hline $\mathrm{N} 1-\mathrm{C} 1-\mathrm{C} 2-\mathrm{C} 3$ & $-178.07(14)$ & $\mathrm{C} 3-\mathrm{C} 4-\mathrm{C} 5-\mathrm{C} 6$ & $2.1(2)$ \\
\hline
\end{tabular}




$\begin{array}{llll}\mathrm{N} 2-\mathrm{C} 6-\mathrm{C} 1-\mathrm{N} 1 & -1.2(2) & \mathrm{C} 3-\mathrm{C} 4-\mathrm{C} 7-\mathrm{O} 3 & 177.40(13) \\ \mathrm{N} 2-\mathrm{C} 6-\mathrm{C} 1-\mathrm{C} 2 & 178.91(14) & \mathrm{C} 7-\mathrm{O} 3-\mathrm{C} 8-\mathrm{O} 4 & 0.1(2) \\ \mathrm{N} 2-\mathrm{C} 6-\mathrm{C} 5-\mathrm{C} 4 & 179.13(13) & \mathrm{C} 7-\mathrm{O} 3-\mathrm{C} 8-\mathrm{C} 9 & 179.98(14) \\ \mathrm{C} 6-\mathrm{C} 1-\mathrm{C} 2-\mathrm{C} 3 & 1.9(2) & \mathrm{C} 7-\mathrm{C} 4-\mathrm{C} 5-\mathrm{C} 6 & -178.49(13) \\ \mathrm{C} 1-\mathrm{C} 6-\mathrm{C} 5-\mathrm{C} 4 & -0.5(2) & \mathrm{C} 7-\mathrm{C} 4-\mathrm{C} 3-\mathrm{C} 2 & 178.86(15) \\ \mathrm{C} 1-\mathrm{C} 2-\mathrm{C} 3-\mathrm{C} 4 & -0.2(2) & \mathrm{C} 8-\mathrm{O} 3-\mathrm{C} 7-\mathrm{C} 4 & -179.68(12)\end{array}$

Hydrogen-bond geometry $\left(\AA,{ }^{\circ}\right)$

\begin{tabular}{lllll}
\hline$D-\mathrm{H} \cdots A$ & $D-\mathrm{H}$ & $\mathrm{H} \cdots A$ & $D \cdots A$ & $D-\mathrm{H} \cdots A$ \\
\hline $\mathrm{N} 2-\mathrm{H} 2 A \cdots \mathrm{O} 4^{\mathrm{i}}$ & $0.83(2)$ & $2.18(2)$ & $3.005(2)$ & $171.5(17)$ \\
$\mathrm{N} 2-\mathrm{H} 2 B \cdots \mathrm{O} 1$ & $0.84(2)$ & $2.06(2)$ & $2.6600(19)$ & $128.0(16)$ \\
$\mathrm{N} 2-\mathrm{H} 2 B \cdots \mathrm{O} 1^{\mathrm{ii}}$ & $0.84(2)$ & $2.44(2)$ & $3.1443(19)$ & $142.7(16)$ \\
\hline
\end{tabular}

Symmetry codes: (i) $-x+3 / 2, y+1 / 2,-z+3 / 2$; (ii) $-x+1 / 2,-y+3 / 2,-z+1$. 